\title{
Global field experiments for potato simulations
}

Rubí Raymundo ${ }^{*}$, Senthold Asseng ${ }^{1}$, Rishi Prasad ${ }^{1}$, Ulrich Kleinwechter ${ }^{2}$, Bruno Condori ${ }^{3}$, Walter Bowen ${ }^{10}$, Joost Wolf $^{4}$, Jørgen E. Olesen ${ }^{5}$, Qiaoxue Dong ${ }^{6}$, Lincoln Zotarelli ${ }^{1}$, Manuel Gastelo ${ }^{3}$, Ashok Alva ${ }^{7}$, Maria Travasso ${ }^{8}$ and Vijay Arora ${ }^{9}$

${ }^{1}$ Agricultural and Biological Engineering Department, University of Florida, Gainesville, Florida, USA.

2 International Institute for Applied Systems Analysis (IIASA), Laxenburg, Austria.

3 International Potato Center (CIP), Lima, Peru.

4 Plant Production Systems Group, Wageningen University, The Netherlands.

${ }^{5}$ Department of Agroecology, Aarhus University, Tjele, Denmark.

${ }^{6}$ China Agricultural University, Beijing, China.

7 United States Department of Agriculture-Agricultural Research Service (USDA-ARS), Sidney, MT.

${ }^{8}$ Instituto de Clima y Agua (INTA), Los Reseros y Las Cabañas, Castelar, Argentina.

${ }^{9}$ Department of Soil Science, Punjab Agricultural University, Ludhiana, India.

10 Institute of Food and Agricultural Sciences (IFAS), University of Florida, Gainesville, Florida, USA.

*e-mail: raymundo@umn.edu

Abstract: A large field potato experimental dataset has been assembled for simulation modeling. The data are from temperate, subtropical, and tropical regions across the world and include 87 experiments with 204 treatments. Treatments include nitrogen fertilizer, irrigation, atmospheric $\mathrm{CO}_{2}$ levels, temperature, cultivars, and locations. For all experiments, measurements include tuber fresh and dry weight. For some experiments, measurements include in-season biomass, leaf area index, stem and leaf weight, $\mathrm{N}$ uptake, soil water, and soil $\mathrm{N}$ contents. Each experiment has soil characteristics and daily data for solar radiation, rainfall, and maximum and minimum temperature. The data have been quality checked and used in a previous simulation exercise. All data are in AGMIP format.

Keywords: potato, field experimental data, SUBSTOR-potato, simulations.

1 EXPERIMENTAL POTATO DATASETS: A set of 87 field experiments with 204 treatments and 32 cultivars, 3 species, and 12 soil types has been assembled. These datasets have been quality checked and used in a previous model performance test of the SUBSTOR-potato model (Raymundo et al., 2017).

Experiments include information on sowing date, harvest date, and detailed treatment descriptions. Some experiments also include emergence date. Treatments include nitrogen fertilizer, irrigation, atmospheric $\mathrm{CO}_{2}$ levels (Open Top Chambers - OTC and Free-Air- $\mathrm{CO}_{2}$-Enrichment - FACE), temperature, cultivars, and locations (Table 1). 


\begin{tabular}{|c|c|c|c|c|c|c|c|c|c|c|c|}
\hline Location & Year & № $\operatorname{tr}^{*}$ & $\begin{array}{c}\text { Sowing } \\
\text { date } \\
\text { DOY }\end{array}$ & $\begin{array}{c}\text { Emergence } \\
\text { date } \\
\text { DOY }\end{array}$ & $\begin{array}{c}\text { Harvest } \\
\text { date } \\
\text { DOY }\end{array}$ & $\begin{array}{c}\mathrm{N} \\
\text { application } \\
\mathrm{kg} \mathrm{ha}^{-1 * \star}\end{array}$ & $\begin{array}{c}\text { Irrigation } \\
\text { mm }\end{array}$ & $\begin{array}{c}\text { Rainfall } \\
\mathrm{mm}\end{array}$ & $\begin{array}{c}\text { Type of } \\
\text { irrigation } \\
-\end{array}$ & $\begin{array}{l}\mathrm{CO}_{2} \\
\mathrm{ppm}\end{array}$ & Cultivar \\
\hline \multirow[t]{4}{*}{ Argentina, Balcarce } & 1991 & 1 & 298 & n.a. & 64 & 0 & 113 & 540 & Mixed & Default & Spunta \\
\hline & & 2 & 298 & n.a. & 64 & $60(1)$ & 113 & 540 & Mixed & Default & Spunta \\
\hline & & 3 & 298 & n.a. & 64 & $120(1)$ & 113 & 540 & Mixed & Default & Spunta \\
\hline & & 4 & 298 & n.a. & 64 & $160(1)$ & 113 & 540 & Mixed & Default & Spunta \\
\hline \multirow[t]{3}{*}{ Australia } & 1970 & 1 & 222 & 273 & 356 & $425(2)$ & n.a. & 219 & Full & Default & Sebago \\
\hline & & 2 & 222 & 273 & 356 & $425(2)$ & n.a. & 219 & Full & Default & Sebago \\
\hline & & 3 & 222 & 273 & 356 & $425(2)$ & n.a. & 219 & Full & Default & Sebago \\
\hline \multirow[t]{6}{*}{ Belgium, Tervuren } & $1998+$ & $1^{\mathrm{N}}$ & 127 & 135 & 257 & $205(2)$ & 373 & 423 & Mixed & 380 & Bintje \\
\hline & & $2^{\mathrm{N}}$ & 127 & 135 & 257 & $205(2)$ & 373 & 423 & Mixed & 386 & Bintje \\
\hline & & $3^{N}$ & 127 & 135 & 257 & $205(2)$ & 373 & 423 & Mixed & 676 & Bintje \\
\hline & $1999+$ & $1^{\mathrm{N}}$ & 131 & 144 & 250 & $220(2)$ & 182 & 204 & Full & 365 & Bintje \\
\hline & & $2^{\mathrm{N}}$ & 131 & 144 & 250 & $220(2)$ & 181 & 204 & Full & 370 & Bintje \\
\hline & & $3^{N}$ & 131 & 144 & 250 & $220(2)$ & 183 & 204 & Mixed & 664 & Bintje \\
\hline \multirow[t]{2}{*}{ Bolivia, Belen } & 1997 & 1 & 288 & 316 & 84 & $110(1)$ & n.a. & 264 & Full & Default & Waycha \\
\hline & & 2 & 288 & 316 & 84 & $110(1)$ & n.a. & 264 & Full & Default & Lucky \\
\hline Bolivia, Chinoli & 1997 & 1 & 301 & 340 & 62 & 124(1) & n.a. & 275 & Full & Default & Desiree \\
\hline \multirow[t]{3}{*}{ Bolivia, Koari } & 1997 & 1 & 281 & 329 & 111 & $100(1)$ & - & 540 & Rainfed & Default & Waycha \\
\hline & & 2 & 281 & 329 & 111 & $100(1)$ & - & 540 & Rainfed & Default & Alpha \\
\hline & & 3 & 281 & 329 & 111 & $100(1)$ & - & 540 & Rainfed & Default & Lucky \\
\hline Bolivia, Patacamaya & 1997 & $1^{\mathrm{N}}$ & 300 & 351 & 112 & $110(1)$ & n.a. & 341 & Full & Default & Waycha \\
\hline & & $2^{\mathrm{N}}$ & 300 & 351 & 112 & $110(1)$ & n.a. & 341 & Full & Default & Lucky \\
\hline Bolivia, Patacamaya & $1998-1$ & 1 & 292 & 347 & 110 & $110(1)$ & n.a. & 334 & Full & Default & Waycha \\
\hline & & 2 & 292 & 347 & 110 & $110(1)$ & n.a. & 334 & Full & Default & Lucky \\
\hline Bolivia, Patacamaya & $1998-2$ & $1^{\mathrm{N}}$ & 292 & 342 & 110 & $110(1)$ & n.a. & 334 & Full & Default & Waycha \\
\hline & & $2^{\mathrm{N}}$ & 292 & 342 & 110 & $110(1)$ & n.a. & 334 & Full & Default & Lucky \\
\hline Bolivia, Toralapa & 1993 & $1^{\mathrm{N}}$ & 295 & 351 & 103 & $120(1)$ & n.a. & 440 & Full & Default & Waycha \\
\hline & & $2^{\mathrm{N}}$ & 295 & 351 & 103 & $120(1)$ & n.a. & 440 & Full & Default & Alpha \\
\hline & & $3^{N}$ & 295 & 351 & 103 & $120(1)$ & n.a. & 440 & Full & Default & Lucky \\
\hline & & $4^{\mathrm{N}}$ & 295 & 351 & 103 & $120(1)$ & - & 440 & Rainfed & Default & Waycha \\
\hline & & $5^{N}$ & 295 & 351 & 103 & $120(1)$ & - & 440 & Rainfed & Default & Alpha \\
\hline & & $6^{\mathrm{N}}$ & 295 & 351 & 103 & $120(1)$ & - & 440 & Rainfed & Default & Lucky \\
\hline China, Huhhot & 1996 & 1 & 118 & n.a. & 250 & $150(2)$ & n.a. & 252 & Full & Default & Desiree \\
\hline China, Huhhot & 1998 & 1 & 115 & n.a. & 253 & $150(2)$ & n.a. & 470.9 & Full & Default & Desiree \\
\hline China, Jining & 1999 & 1 & 119 & n.a. & 234 & $74(2)$ & n.a. & 156 & Full & Default & Desiree \\
\hline & & 2 & 119 & n.a. & 234 & $74(2)$ & n.a. & 156 & Full & Default & Kexin 1 \\
\hline & & 3 & 119 & n.a. & 234 & $74(2)$ & n.a. & 156 & Full & Default & Jinguan \\
\hline China, Zhalan & 1997 & 1 & 119 & n.a. & 253 & $180(2)$ & n.a. & 89 & Full & Default & Desiree \\
\hline China, Zhalan & 1998 & 1 & 119 & n.a. & 242 & $90(2)$ & - & 754 & Rainfed & Default & Kexin 1 \\
\hline & & 2 & 119 & n.a. & 242 & $90(2)$ & - & 754 & Rainfed & Default & Neishu 7 \\
\hline Colombia, Cundinamarca & 1999 & 1 & 119 & 143 & 262 & $100(1)$ & - & 392 & Rainfed & Default & Capiro \\
\hline Denmark, Jyndevad & 1981 & 1 & 119 & 149 & 225 & 155(1) & 169 & 431 & Mixed & Default & Bintje \\
\hline & 1982 & 1 & 119 & 148 & 236 & $155(1)$ & 222 & 521 & Mixed & Default & Bintje \\
\hline & 1983 & 1 & 122 & 148 & 215 & $155(1)$ & 156 & 370 & Mixed & Default & Bintje \\
\hline Denmark, Jyndevad & 1990 & 1 & 107 & n.a. & 267 & $180(1)$ & 107 & 499 & Mixed & Default & Bintje \\
\hline & & 2 & 107 & n.a. & 267 & $180(4)$ & 104 & 499 & Mixed & Default & Bintje \\
\hline & & 3 & 107 & n.a. & 267 & $180(4)$ & 105 & 499 & Mixed & Default & Bintje \\
\hline & 1991 & 1 & 101 & 149 & 273 & $180(1)$ & 137 & 390 & Mixed & Default & Bintje \\
\hline & & 2 & 101 & 149 & 273 & $180(3)$ & 137 & 390 & Mixed & Default & Bintje \\
\hline & & 3 & 101 & 149 & 273 & $180(3)$ & 137 & 390 & Mixed & Default & Bintje \\
\hline & 1992 & 1 & 100 & 139 & 224 & $180(1)$ & 231 & 195 & Mixed & Default & Bintje \\
\hline & & 2 & 100 & 139 & 224 & $180(3)$ & 231 & 195 & Mixed & Default & Bintje \\
\hline & & 3 & 100 & 139 & 224 & $180(4)$ & 231 & 195 & Mixed & Default & Bintje \\
\hline & 1993 & 1 & 110 & 133 & 263 & $180(1)$ & 70 & 350 & Mixed & Default & Bintje \\
\hline & & 2 & 110 & 133 & 263 & $180(3)$ & 70 & 350 & Mixed & Default & Bintje \\
\hline & & 3 & 110 & 133 & 263 & $180(4)$ & 70 & 350 & Mixed & Default & Bintje \\
\hline Denmark, Jyndevad & 1984 & 1 & 117 & 151 & 278 & $150(1)$ & 71 & 519 & Mixed & Default & Tilva \\
\hline & & 2 & 117 & 151 & 278 & $200(1)$ & 71 & 519 & Mixed & Default & Tilva \\
\hline & 1985 & 1 & 119 & 149 & 270 & 150(1) & 27 & 465 & Mixed & Default & Tilva \\
\hline & & 2 & 119 & 149 & 270 & $200(1)$ & 27 & 465 & Mixed & Default & Tilva \\
\hline & 1986 & 1 & 118 & 146 & 293 & 150(1) & 186 & 488 & Mixed & Default & Tilva \\
\hline & & 2 & 118 & 146 & 293 & $200(1)$ & 186 & 488 & Mixed & Default & Tilva \\
\hline Denmark, Tylstrup & 1981 & 1 & 120 & 155 & 272 & $140(1)$ & 25 & 405 & Mixed & Default & Bintje \\
\hline & & 2 & 120 & 152 & 272 & $140(1)$ & 25 & 405 & Mixed & Default & Sava \\
\hline & & 3 & 120 & 149 & 272 & $140(1)$ & 25 & 405 & Mixed & Default & Posmo \\
\hline & & 4 & 120 & 150 & 272 & $140(1)$ & 25 & 405 & Mixed & Default & Kaptah \\
\hline & & 5 & 120 & 145 & 272 & $140(1)$ & 25 & 405 & Mixed & Default & Dianella \\
\hline & 1982 & 1 & 109 & 149 & 270 & 180(1) & 185 & 607 & Mixed & Default & Bintje \\
\hline & & 2 & 109 & 155 & 270 & 180(1) & 185 & 607 & Mixed & Default & Sava \\
\hline & & 3 & 109 & 152 & 270 & 180(1) & 185 & 607 & Mixed & Default & Posmo \\
\hline & & 4 & 109 & 152 & 270 & $180(1)$ & 185 & 607 & Mixed & Default & Kaptah \\
\hline & & 5 & 109 & 149 & 298 & 180(1) & 185 & 607 & Mixed & Default & Dianella \\
\hline & 1983 & 1 & 109 & 154 & 298 & $160(2)$ & 135 & 566 & Mixed & Default & Bintje \\
\hline & & 2 & 109 & 154 & 298 & $160(2)$ & 135 & 566 & Mixed & Default & Sava \\
\hline & & 3 & 109 & 150 & 298 & $160(2)$ & 135 & 566 & Mixed & Default & Posmo \\
\hline & & 4 & 109 & 154 & 298 & $160(2)$ & 135 & 566 & Mixed & Default & Kaptah \\
\hline
\end{tabular}




\begin{tabular}{|c|c|c|c|c|c|c|c|c|c|c|c|}
\hline Location & Year & № tr* & $\begin{array}{c}\text { Sowing } \\
\text { date } \\
\text { DOY }\end{array}$ & $\begin{array}{c}\text { Emergence } \\
\text { date } \\
\text { DOY }\end{array}$ & $\begin{array}{c}\text { Harvest } \\
\text { date } \\
\text { DOY }\end{array}$ & $\begin{array}{c}\mathrm{N} \\
\text { application } \\
\mathrm{kg} \mathrm{ha}^{-1 \star \star}\end{array}$ & $\begin{array}{c}\text { Irrigation } \\
\mathrm{mm}\end{array}$ & $\begin{array}{c}\text { Rainfall } \\
\mathrm{mm}\end{array}$ & $\begin{array}{c}\text { Type of } \\
\text { irrigation } \\
-\end{array}$ & $\begin{array}{l}\mathrm{CO}_{2} \\
\mathrm{ppm}\end{array}$ & Cultivar \\
\hline Denmark, Tylstrup & 1983 & 5 & 109 & 149 & 298 & $160(2)$ & 135 & 566 & Mixed & Default & Dianella \\
\hline \multirow[t]{4}{*}{ Ecuador, San Gabriel } & 1985 & 1 & 45 & 76 & 241 & $168(2)$ & - & 426 & Rainfed & Default & INIAP-maria \\
\hline & & 2 & 45 & 76 & 241 & $117(1)$ & - & 426 & Rainfed & Default & INIAP-gabriela \\
\hline & & 3 & 45 & 76 & 241 & $168(2)$ & - & 426 & Rainfed & Default & INIAP-maria \\
\hline & & 4 & 45 & 76 & 241 & $117(1)$ & - & 426 & Rainfed & Default & INIAP-gabriela \\
\hline \multirow[t]{2}{*}{ Finland, Jokioinen } & $1998+$ & 1 & 152 & 163 & 270 & $80(2)$ & - & 329 & Full & 375 & Bintje \\
\hline & $1999+$ & 1 & 149 & 159 & 264 & $80(2)$ & - & 122 & Full & 550 & Bintje \\
\hline \multirow[t]{6}{*}{ Germany, Giessen } & $1998+$ & $1^{\mathrm{N}}$ & 124 & 134 & 250 & $150(2)$ & 67 & 365 & Mixed & 373 & Bintje \\
\hline & & $2^{\mathrm{N}}$ & 124 & 134 & 250 & $150(2)$ & 67 & 365 & Mixed & 541 & Bintje \\
\hline & & $3^{N}$ & 124 & 134 & 250 & $150(2)$ & 67 & 365 & Mixed & 690 & Bintje \\
\hline & $1999+$ & $1^{\mathrm{N}}$ & 130 & 147 & 258 & $116(2)$ & 152 & 267 & Full & 380 & Bintje \\
\hline & & $2^{\mathrm{N}}$ & 130 & 147 & 258 & $116(2)$ & 152 & 267 & Full & 541 & Bintje \\
\hline & & $3^{N}$ & 130 & 147 & 258 & $116(2)$ & 148 & 267 & Full & 708 & Bintje \\
\hline \multirow[t]{4}{*}{ Germany, Giessen } & 1998++ & $1^{\mathrm{N}}$ & 125 & 135 & 257 & $150(2)$ & 186 & 417 & Mixed & 401 & Bintje \\
\hline & & $2^{N}$ & 125 & 135 & 257 & $150(2)$ & 186 & 417 & Mixed & 429 & Bintje \\
\hline & 1999++ & 1 & 130 & 145 & 239 & $156(2)$ & 128 & 250 & Full & 374 & Bintje \\
\hline & & 2 & 130 & 145 & 239 & $156(2)$ & 126 & 250 & Full & 491 & Bintje \\
\hline \multirow[t]{5}{*}{ India, Ludhiana } & 2008 & 1 & 290 & n.a. & 29 & 0 & 80 & 18 & Mixed & Default & Kufri Bahar \\
\hline & & 2 & 290 & n.a. & 29 & $136(2)$ & 80 & 18 & Mixed & Default & Kufri Bahar \\
\hline & & 3 & 290 & n.a. & 29 & $180(2)$ & 80 & 18 & Mixed & Default & Kufri Bahar \\
\hline & & 4 & 290 & n.a. & 29 & $224(2)$ & 80 & 18 & Mixed & Default & Kufri Bahar \\
\hline & & 5 & 290 & n.a. & 29 & 0 & 160 & 18 & Mixed & Default & Kufri Bahar \\
\hline India, Ludhiana & 2008 & 6 & 290 & n.a. & 29 & $136(2)$ & 160 & 18 & Mixed & Default & Kufri Bahar \\
\hline & & 7 & 290 & n.a. & 29 & $180(2)$ & 160 & 18 & Mixed & Default & Kufri Bahar \\
\hline & & 8 & 290 & n.a. & 29 & $224(2)$ & 160 & 18 & Mixed & Default & Kufri Bahar \\
\hline & & 9 & 290 & n.a. & 29 & 0 & 200 & 18 & Mixed & Default & Kufri Bahar \\
\hline & & 10 & 290 & n.a. & 29 & $136(2)$ & 200 & 18 & Mixed & Default & Kufri Bahar \\
\hline & & 11 & 290 & n.a. & 29 & $180(2)$ & 200 & 18 & Mixed & Default & Kufri Bahar \\
\hline & & 12 & 290 & n.a. & 29 & $224(2)$ & 200 & 18 & Mixed & Default & Kufri Bahar \\
\hline & 2010 & 1 & 285 & n.a. & 29 & 0 & 80 & 32 & Mixed & Default & Kufri Bahar \\
\hline & & 2 & 285 & n.a. & 29 & $136(2)$ & 80 & 32 & Mixed & Default & Kufri Bahar \\
\hline & & 3 & 285 & n.a. & 29 & $180(2)$ & 80 & 32 & Mixed & Default & Kufri Bahar \\
\hline & & 4 & 285 & n.a. & 29 & $224(2)$ & 80 & 32 & Mixed & Default & Kufri Bahar \\
\hline & & 5 & 285 & n.a. & 29 & 0 & 160 & 32 & Mixed & Default & Kufri Bahar \\
\hline & & 6 & 285 & n.a. & 29 & $136(2)$ & 160 & 32 & Mixed & Default & Kufri Bahar \\
\hline & & 7 & 285 & n.a. & 29 & $180(2)$ & 160 & 32 & Mixed & Default & Kufri Bahar \\
\hline & & 8 & 285 & n.a. & 29 & $224(2)$ & 160 & 32 & Mixed & Default & Kufri Bahar \\
\hline & & 9 & 285 & n.a. & 29 & 0 & 200 & 32 & Mixed & Default & Kufri Bahar \\
\hline & & 10 & 285 & n.a. & 29 & $136(2)$ & 200 & 32 & Mixed & Default & Kufri Bahar \\
\hline & & 11 & 285 & n.a. & 29 & $180(2)$ & 200 & 32 & Mixed & Default & Kufri Bahar \\
\hline & & 12 & 285 & n.a. & 29 & $224(2)$ & 200 & 32 & Mixed & Default & Kufri Bahar \\
\hline India, Modipuran & 2002 & 1 & 288 & n.a. & 3 & 181(2) & n.a. & 0 & Full & Default & Kufri Bahar \\
\hline & 2003 & 1 & 299 & n.a. & 18 & 181(2) & n.a. & 30 & Full & Default & Kufri Bahar \\
\hline & 2004 & 1 & 293 & n.a. & 11 & $181(2)$ & n.a. & 0 & Full & Default & Kufri Bahar \\
\hline & 2005 & 1 & 289 & n.a. & 8 & $181(2)$ & n.a. & 1 & Full & Default & Kufri Bahar \\
\hline & 2006 & 1 & 294 & n.a. & 13 & $181(2)$ & n.a. & 0 & Full & Default & Kufri Bahar \\
\hline & 2007 & 1 & 298 & n.a. & 17 & $181(2)$ & n.a. & 0 & Full & Default & Kufri Bahar \\
\hline & 2008 & 1 & 290 & n.a. & 8 & $181(2)$ & n.a. & 12 & Full & Default & Kufri Bahar \\
\hline & 2009 & 1 & 302 & n.a. & 21 & $181(2)$ & n.a. & 0 & Full & Default & Kufri Bahar \\
\hline Ireland, Carlow & 1998 & $1^{\mathrm{N}}$ & 128 & 138 & 280 & $250(2)$ & 61 & 343 & Full & 372 & Bintje \\
\hline & & $2^{N}$ & 128 & 138 & 280 & $250(2)$ & 31 & 343 & Full & 693 & Bintje \\
\hline & 1999 & $1^{\mathrm{N}}$ & 140 & 152 & 249 & $250(2)$ & 89 & 392 & Full & 372 & Bintje \\
\hline & & $2^{\mathrm{N}}$ & 140 & 152 & 249 & $250(2)$ & 91 & 392 & Full & 670 & Bintje \\
\hline Italy, Rapolano & 1998++ & 1 & 141 & 149 & 237 & $240(2)$ & 309 & 554 & Full & 366 & Bintje \\
\hline & & 2 & 141 & 149 & 237 & $240(2)$ & 294 & 554 & Full & 552 & Bintje \\
\hline & & 3 & 141 & 149 & 237 & $240(2)$ & 285 & 554 & Full & 367 & Bintje \\
\hline & 1999++ & 1 & 126 & 147 & 237 & $250(2)$ & 462 & 146 & Mixed & 367 & Bintje \\
\hline & & 2 & 126 & 147 & 237 & $250(2)$ & 462 & 146 & Mixed & 552 & Bintje \\
\hline & & 3 & 126 & 147 & 237 & $250(2)$ & 462 & 146 & Mixed & 367 & Bintje \\
\hline Peru, La Molina & 2003 & 1 & 181 & n.a. & 314 & $310(2)$ & 450 & 0 & Irrigated & Default & Amarilis \\
\hline & 2004 & 1 & 177 & n.a. & 307 & $310(2)$ & 350 & 0 & Irrigated & Default & Amarilis \\
\hline & 2005 & 1 & 164 & n.a. & 281 & $298(2)$ & 450 & 0 & Irrigated & Default & Amarilis \\
\hline & 2006 & 1 & 186 & n.a. & 319 & $310(2)$ & 350 & 0 & Irrigated & Default & Amarilis \\
\hline & 2007 & 1 & 152 & n.a. & 275 & $350(2)$ & 400 & 0 & Irrigated & Default & Amarilis \\
\hline & 2008 & 1 & 182 & n.a. & 294 & $235(2)$ & 350 & 0 & Irrigated & Default & Amarilis \\
\hline & 2009 & 1 & 183 & n.a. & 292 & $235(2)$ & 400 & 0 & Irrigated & Default & Amarilis \\
\hline & 2010 & 1 & 196 & n.a. & 307 & $227(2)$ & 350 & 0 & Irrigated & Default & Amarilis \\
\hline Peru, La Molina & 2013 & 1 & 179 & 200 & 288 & $210(2)$ & 215 & 14 & Full & Default & Achirana \\
\hline & & 2 & 179 & 198 & 288 & $210(2)$ & 215 & 14 & Full & Default & Atlantic \\
\hline & & 3 & 179 & 198 & 288 & $210(2)$ & 215 & 14 & Full & Default & Sarnav \\
\hline Peru, La Molina & 1985 & 1 & 30 & 60 & 122 & $160(2)$ & 449 & 3 & Irrigated & Default & DTO-33 \\
\hline & & 2 & 30 & 60 & 130 & $160(2)$ & 480 & 3 & Irrigated & Default & LT1 \\
\hline & & 3 & 30 & 60 & 144 & $160(2)$ & 485 & 3 & Irrigated & Default & Revolucion \\
\hline & & 4 & 176 & 206 & 285 & $160(2)$ & 315 & 1 & Irrigated & Default & DTO-33 \\
\hline & & 5 & 176 & 206 & 291 & $160(2)$ & 257 & 1 & Irrigated & Default & LT1 \\
\hline
\end{tabular}


Raymundo et al. 2018, Open Data Journal for Agricultural Research, vol. 4, pg. 35-44

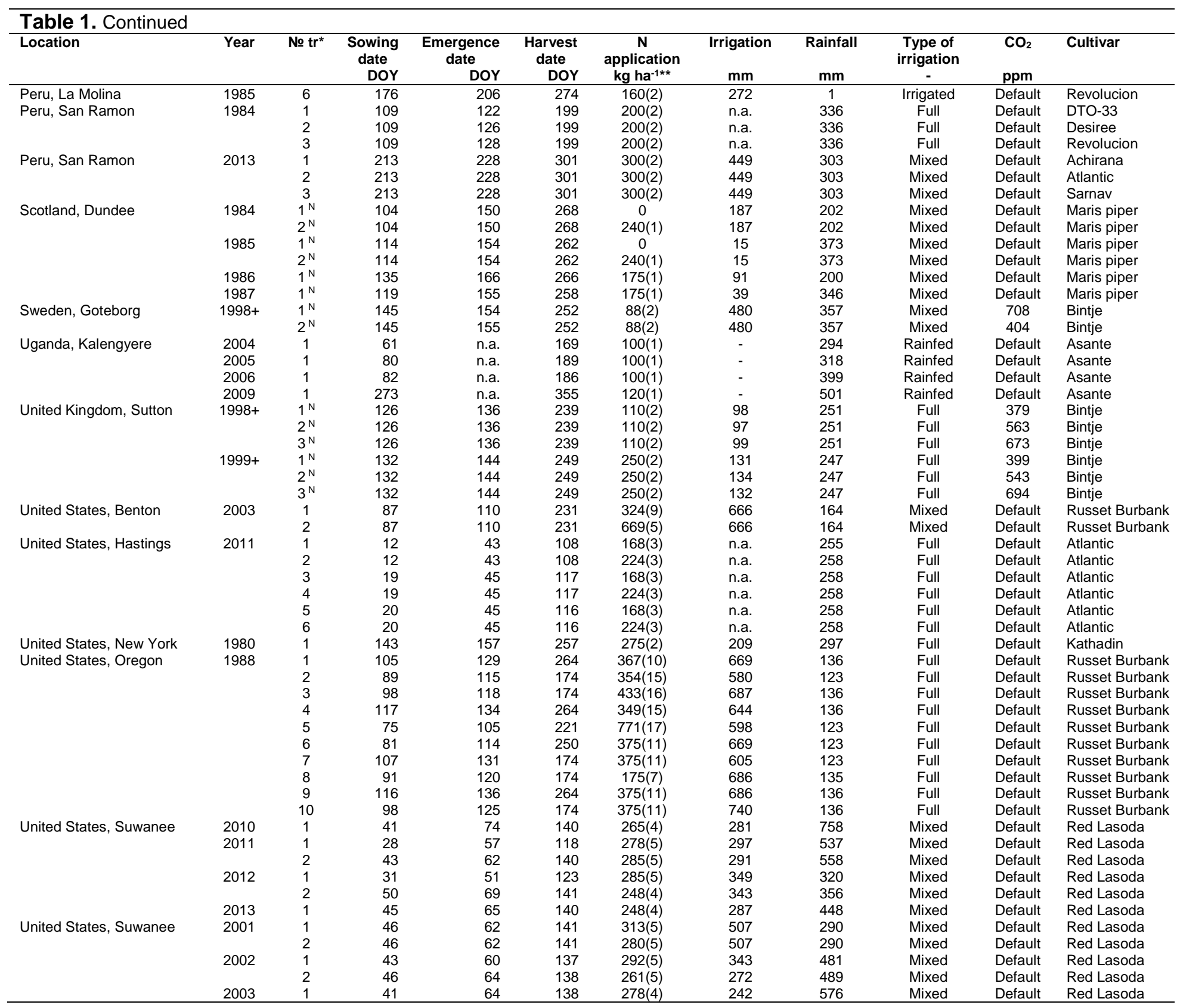

$\mathrm{N} \quad$ Treatments with estimated initial soil $\mathrm{N}$

* Number or treatments.

** Number of nitrogen applications are in parenthesis.

$+\quad$ OTC: Open Top Chambers

$++\quad$ FACE: Free-Air-CO2-Enrichment, OTC: Open Top Chambers

n.a. Not available.

Not applicable.

Full Automatic irrigation.

Default Atmospheric $\mathrm{CO}_{2}$ concentration was for any year used from Mauna Loa Observatory, Hawaii (Bacastow et al., 1985).

Measurements include tuber fresh and dry weight for all experiments. For some experiments, measurements also include biomass, leaf area index, stem and leaf weight, $\mathrm{N}$ uptake, soil water, and soil $\mathrm{N}$ contents (Table 2). Most experiments had in-season measurements (more than two measurements), whereas 21 experiments only had information of final tuber yield. Across all experiments a total of 14 measured variables are provided. Experiments without reference have not been published before (Table 2). 


\begin{tabular}{|c|c|c|c|c|c|c|c|c|c|c|c|}
\hline Location & Year & lat $^{\mathrm{a}}$ & $\operatorname{lon}^{b}$ & alt $^{\mathrm{c}}$ & Objective $^{d}$ & $\operatorname{tr}^{\mathrm{e}}$ & cult $^{t}$ & $\begin{array}{r}\text { In-season } \\
\text { sampling }\end{array}$ & Measured variables $^{h}$ & Soil Type & Reference \\
\hline Argentina, Balcarce & 1991 & -37.8 & -58.3 & 97 & $\mathrm{~N}$ rates & 4 & 1 & 5 & tu & $\mathrm{CL}$ & (Travasso et al., 1996) \\
\hline Australia, & 1970 & -35.0 & 149.0 & & Radiation deficit & 3 & 1 & 10 & tuf,LAI & SC & (Hoogenboom et al., 2015) \\
\hline Belgium, Tervuren & 1998-1999 & 50.8 & 4.5 & 97 & $\mathrm{CO}_{2} \mathrm{OTC}$ & 1 & 1 & 2 & tu,le,st,to,LAl & SiL & (De Temmerman et al., 2002) \\
\hline Bolivia, Belen & 1997 & -16.0 & -68.7 & 3640 & Cultivar adaptation & 1 & 3 & 3 & tu,le & $\mathrm{CL}$ & (Condori et al., 2010) \\
\hline Bolivia, Chinoli & 1997 & -19.6 & -65.3 & 3450 & Cultivar adaptation & 1 & 1 & 4 & tu,le,st,ro,to & SL & (Condori et al., 2010) \\
\hline Bolivia, Koari & 1997 & -17.4 & -65.6 & 3500 & Cultivar adaptation & 1 & 3 & 3 & tu,le,st & $\mathrm{CL}$ & (Condori et al., 2010) \\
\hline Bolivia, Patacamaya & 1997 & -17.2 & -68.0 & 3780 & Cultivar adaptation & 1 & 2 & 3-4 & tu,le,st,ro & $\mathrm{SiCL}$ & (Condori et al., 2010) \\
\hline Bolivia, Patacamaya & 1998 (2) & -17.2 & -68.0 & 3780 & Cultivar adaptation & 1 & 2 & 3-4 & tu,le,st,ro & $\mathrm{SCL}$ & (Condori et al., 2010) \\
\hline Bolivia, Toralapa & 1993 & -17.5 & -65.7 & 3430 & Cultivar adaptation & 2 & 3 & 7 & tu,le,st,ro, & CL & (Condori et al., 2010) \\
\hline China, Huhhot & 1996 & 40.5 & 111.4 & 1065 & Partitioning & 1 & 1 & 6 & tu, LAl & SL & (Gao et al., 2003) \\
\hline China, Huhhot & 1998 & 40.5 & 111.4 & 1065 & Partitioning & 1 & 2 & 6 & tu,le,st,to & SL & (Liu et al., 2003b) \\
\hline China, Jining & 1999 & 41.0 & 113.0 & & Partitioning & 3 & 3 & 6 & tu,le,st,to & SL & (Gao et al., 2004) \\
\hline China, Zhalan & 1997 & 48.0 & 123.0 & & Various & 1 & 1 & 7 & tu,le,st,to & SL & (Liu et al., 2003a) \\
\hline China, Zhalan & 1998 & 48.0 & 123.0 & & Partitioning & 2 & 2 & 7 & tu,le,st,to & SL & (Gao et al., 2004) \\
\hline Colombia, Cundinamarca & 1999 & 4.4 & -74.1 & & Model validation & 1 & 1 & 9 & tu,le,st,to,LAl & SiL & $\begin{array}{r}\text { (Forero Hernandez and Garzon } \\
\text { Montaño, 2000) }\end{array}$ \\
\hline Denmark, Jyndevad & $1982-1983$ & 54.9 & 9.1 & 10 & Various & 3 & 4 & $5-9$ & tu,to & $\mathrm{s}$ & (Jørgensen, 1984) \\
\hline Denmark, Jyndevad & $1990-1993$ & 54.9 & 9.1 & 10 & $\mathrm{~N}$ rates & 4 & 1 & $6-7$ & tu,ro,to,tuN,roN,toN & s & (Edlefsen, 1991) \\
\hline Denmark, Jyndevad & $1984-1986$ & 54.9 & 9.1 & 10 & $\mathrm{~N}$ rates & 2 & 1 & $14-16$ & tu,to,tuN & s & (Jørgensen and Edlefsen, 1987) \\
\hline Denmark, Tylstrup & $1981-1983$ & 57.2 & 10.0 & 10 & Various & 4 & 4 & $13-14$ & tu,to & s & (Bach and Nielsen, 1985) \\
\hline Ecuador, San Gabriel & 1985 & 0.6 & -77.8 & & $\mathrm{~N}$ rates & 3 & 2 & 4 & tu & SL & (Clavijo Ponce, 1999) \\
\hline Finland, Jokioinen & 1998-1999 & 60.8 & 23.5 & 84 & $\mathrm{CO}_{2} \mathrm{OTC}$ & 3 & 1 & 2 & tu,le,st & SL & (De Temmerman et al., 2002) \\
\hline Germany, Giessen & 1998-1999 & 50.6 & 8.7 & 68 & $\mathrm{CO}_{2} \mathrm{OTC}$ & 1 & 1 & 2 & tu,le,st,to,LAl & SL & (De Temmerman et al., 2002) \\
\hline Germany, Giessen & 1998-1999 & 50.6 & 8.7 & 68 & $\mathrm{CO}_{2} \mathrm{FACE}$ & 1 & 1 & 2 & tu,le,st,to,LAl & SL & (De Temmerman et al., 2002) \\
\hline India, Ludhiana & 2008-2011 & 30.9 & 75.8 & 244 & $\mathrm{~N}$ rates and irrigation & 4 & 1 & 1 & tuf & SL & (Arora et al., 2013) \\
\hline India, Modipuran & 2002-2009 & 28.7 & 77.2 & 228 & Various & 1 & 1 & 1 & tuf & L,SiCL & (Kleinwechter et al., 2016) \\
\hline Ireland, Carlow & 1998-1999 & 52.9 & -6.9 & 57 & $\mathrm{CO}_{2} \mathrm{OTC}$ & 1 & 1 & 2 & tu,le,st,to & $\mathrm{SiC}$ & (De Temmerman et al., 2002) \\
\hline Italy, Rapolano & 1998-1999 & 42.7 & 11.9 & 38 & $\mathrm{CO}_{2} \mathrm{FACE}$ & 1 & 1 & 2 & tu,le,st,to,LAl & SL & (De Temmerman et al., 2002) \\
\hline Peru, La Molina & 2003-2010 & -12.1 & -77.0 & 244 & Cultivar adaptation & 1 & 1 & 1 & tuf & SL & (Kleinwechter et al., 2016) \\
\hline Peru, La Molina & 2013 & -12.1 & -77.0 & 244 & Cultivar adaptation & 1 & 3 & 5 & tu,le,st & SL & \\
\hline Peru, La Molina & 1985 & -12.1 & -77.0 & 244 & Cultivar adaptation & 2 & 3 & 5 & tu,le,st,to,LAl & SL, SCL,SL & (Trebejo and Midmore, 1990) \\
\hline Peru, San Ramon & 1984 & -11.1 & -75.3 & 800 & High temperature tolerance & 1 & 3 & 4 & tu,le,st,to & SL & (Nelson, 1987) \\
\hline Peru, San Ramon & 2013 & -11.1 & -75.3 & 800 & High temperature tolerance & 1 & 3 & 5 & tu,le,st,to & SL & \\
\hline Scotland, Dundee & 1984-1985 & 56.5 & -3.1 & 40 & $\mathrm{~N}$ rates & 2 & 1 & 8 & tu,le,st,to,LAl,de,tuN,leN,stN,toN & SL & $\begin{array}{r}\text { (Marshall and Van Den Broek, } \\
1995)\end{array}$ \\
\hline Scotland, Dundee & 1986-1987 & 56.5 & -3.1 & 40 & Irrigation & 4 & 1 & 8 & tu,le,st,to,LAI,SWC & SL & $\begin{array}{r}\text { (Marshall and Van Den Broek, } \\
1995)\end{array}$ \\
\hline Sweden, Gotenborg & 1998 & 57.9 & 12.4 & 58 & $\mathrm{CO}_{2} \mathrm{OTC}$ & 3 & 1 & 2 & tu,le,st,to & SL & (De Temmerman et al., 2002) \\
\hline Uganda, Kalengyere & 2004-2009 & -1.2 & 29.8 & 2400 & Various & 1 & 1 & 1 & tuf & C & (Kleinwechter et al., 2016) \\
\hline United Kingdom, Sutton & 1998-1999 & 52.8 & -1.3 & 87 & $\mathrm{CO}_{2} \mathrm{OTC}$ & 1 & 1 & 2 & tu,le,st,to,LAl & SL & (De Temmerman et al., 2002) \\
\hline United States, Benton & 2003 & 45.9 & -119.5 & & $\mathrm{~N}$ rates & 2 & 1 & $5-6$ & tu,le,st,to,LAl,tuN,leN,stN,toN & $\mathrm{s}$ & (Alva et al., 2010) \\
\hline United States, Hastings & 2011 & 29.7 & -81.5 & 2 & $\mathrm{~N}$ rates & 2 & 3 & 1 & tu,le,st,tuN,leN,stN,toN,soN & $\mathrm{s}$ & (Zotarelli et al., 2014) \\
\hline United States, New York & 1980 & 42.4 & -76.5 & & Various & 1 & 1 & 5 & tu,le,st,to & SL & (Hoogenboom et al., 2015) \\
\hline United States, Oregon & 1988 & 45.8 & -119.3 & & Various & 10 & & $9-15$ & tu,le,st,to,LAl & SL & (Hoogenboom et al., 2015) \\
\hline
\end{tabular}




\begin{tabular}{|c|c|c|c|c|c|c|c|c|c|c|c|}
\hline Location & Year & lat $^{\mathrm{a}}$ & $\operatorname{lon}^{\mathrm{b}}$ & alt $^{c}$ & Objective $^{d}$ & $\operatorname{tr}^{\mathrm{e}}$ & cult $^{f}$ & $\begin{array}{c}\text { In-season } \\
\text { sampling }\end{array}$ & Measured variables $^{h}$ & Soil Type & Reference \\
\hline United States, Suwanee & $2010-2013$ & 30.1 & -83.1 & 13.7 & $\mathrm{~N}$ mass balance & 1 & 1 & 1 & tuf & $\mathrm{s}$ & (Prasad et al., 2015) \\
\hline United States, Suwanee & 2001 & 30.1 & -83.1 & 13.7 & $\mathrm{~N}$ mass balance & 1 & 1 & $3-5$ & tu,le,st,soN,SWC & $\mathrm{s}$ & (Albert, 2002) \\
\hline
\end{tabular}

\section{${ }^{a}$ Latitude.}

${ }^{\mathrm{b}}$ Longitude.

${ }^{c}$ Altitude.

dFACE: Free-Air- $\mathrm{CO}_{2}$-Enrichment, OTC: Open Top Chambers.

e number of treatments.

inumber of cultivars.

g sampling repetitions.

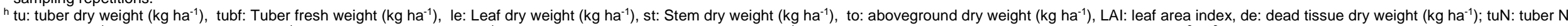
uptake $\left(\mathrm{kg} \mathrm{ha}^{-1}\right)$; le: Leaf $\mathrm{N}$ uptake $\left(\mathrm{kg} \mathrm{ha}^{-1}\right)$; stN: Stem $\mathrm{N}$ uptake $\left(\mathrm{kg} \mathrm{ha}^{-1}\right)$, toN: aboveground nitrogen uptake $\left(\mathrm{kg} \mathrm{ha}^{-1}\right)$, SoN: Soil $\mathrm{N}$ content (ppm), SWC: Soil water content $\left(\mathrm{m}^{3} \mathrm{~m}^{-3}\right)$.

i C: clay; S:sand; Si: silt; L:loam.

All experimental data were organized using the ICASA data dictionary (White et al., 2013) and AgMIP harmonized format (Table 3). Corresponding weather data and soil files are indicated for each experiment. Detailed information of a treatment (FACE and OTC) was given when weather files differed at an experimental site. Weather data include daily solar radiation, maximum and minimum temperatures, and precipitation. Soil profiles were obtained from three sources: 1 ) the original publication; 2) generic soil profiles based on the soil texture from reference; or 3) they were computed using the Sbuild tool embodied in the DSSAT-CSM (Hoogenboom et al., 2015). Methods to compute initial values are explained in (Raymundo et al., 2017). Initial soil conditions were missing in about 20\% of the experiments. In these cases, initial mineral soil $\mathrm{N}$ was chosen by trial and error to closely reproduce one of the treatments (e.g. for $\mathrm{N}$ rate experiments usually the lowest $\mathrm{N}$ treatment). This initial $\mathrm{N}$ amount was then applied to all other treatments in this experiment. (Ritchie et al., 1995). 
Table 3. Potato field experimental files for simulation modeling in AgMIP format.

\begin{tabular}{|c|c|c|c|c|c|c|}
\hline Location & Year & Treatment $^{\mathrm{a}}$ & Weather File & Soil Profile & Soil File & $\begin{array}{c}\text { Management/measurement } \\
\text { File }\end{array}$ \\
\hline Argentina, Balcarce & 1991 & All & INBA9102.WTH & INBA000001 & IN.SOL & INBA9102_mng_fdm.xIsx \\
\hline Australia, & 1970 & All & AUCB0001.WTH & 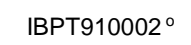 & SOIL.SOL & AUCB0001_mng_fdm.xlsx \\
\hline \multirow{6}{*}{ Belgium, Tervuren } & 1998 & OTC_NF_NF_98 & BE019801.WTH & CHBE000019 $^{\mathrm{g}}$ & CH.SOL & CHBE9801_mng_fdm.xlsx \\
\hline & 1998 & OTC_NF++_NF_98 & BE019801.WTH & CHBE000019 & CH.SOL & CHBE9801_mng_fdm.xIsx \\
\hline & 1998 & AA & BE039801.WTH & CHBE000019 & $\mathrm{CH} . \mathrm{SOL}$ & CHBE9801_mng_fdm.xIsx \\
\hline & 1999 & OTC_NF_NF_99 & BE029901.WHT & CHBE000019 & $\mathrm{CH} . \mathrm{SOL}$ & CHBE9801_mng_fdm.xlsx \\
\hline & 1999 & OTC_NF++_NF_99 & BE029901.WHT & CHBE000019 & CH.SOL & CHBE9801_mng_fdm.xIsx \\
\hline & 1999 & AA & BE049901.WTH & CHBE000019 & CH.SOL & CHBE9801_mng_fdm.xIsx \\
\hline Bolivia, Belen & 1997 & All & PRBE9701.WTH & PRTO9300019 & PR.SOL & PRBE9701_mng_fdm.xIsx \\
\hline Bolivia, Chinoli & 1997 & All & PRCH9701.WTH & IB00000008 & SOIL.SOL & PRCH9701_mng_fdm.xIsx \\
\hline Bolivia, Koari & 1997 & All & PRKO9801.WTH & PRTO9300019 & PR.SOL & PRKO9801_mng_fdm.xIsx \\
\hline Bolivia, Patacamaya -1 & 1997 & All & PRPA9701.WTH & PRPA9800019 & PR.SOL & PRPA9701_mng_fdm.xIsx \\
\hline Bolivia, Patacamaya - 2 & 1998 & All & PRPT9801.WTH & PRPA9800019 & PR.SOL & PRPT9801_mng_fdm.xIsx \\
\hline Bolivia, Patacamaya - 3 & 1998 & All & PRPT9802.WTH & PRPA9800019 & PR.SOL & PRPT9802_mng_fdm.xIsx \\
\hline Bolivia, Toralapa & 1993 & All & PRTO9301.WTH & PRTO9300019 & PR.SOL & PRTO9301_mng_fdm.xIsx \\
\hline China, Huhhot & 1996 & All & AUHU9601.WTH & IB00000009g & $\mathrm{CH} . \mathrm{SOL}$ & AUHU9601_mng_fdm.xIsx \\
\hline China, Huhhot & 1998 & All & AUHU9801.WTH & IB00000009g & $\mathrm{CH} . \mathrm{SOL}$ & AUHU9801_mng_fdm.xlsx \\
\hline China, Jining & 1999 & All & IAWM9901.WTH & IB00000009g & SOIL.SOL & IAWM9901_mng_fdm.xIsx \\
\hline China, Zhalan & 1997 & All & IAHM9701.WTH & IB00000009g & SOIL.SOL & IAHM9701_mng_fdm.xIsx \\
\hline China, Zhalan & 1998 & All & IAHM9801.WTH & IB00000009g & SOIL.SOL & IAHM9801_mng_fdm.xIsx \\
\hline Colombia, Cundinamarca & 1999 & All & ANMG9901.WTH & 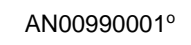 & AN.SOL & ANMG9901_mng_fdm.xIsx \\
\hline \multirow[t]{2}{*}{ Denmark, Jyndevad } & 1982 & All & DIJY8201.WTH & DIJY000002 & DI.SOL & DNJY8201_mng_fdm.xIsx \\
\hline & 1983 & All & DIJY8301.WTH & DIJY000002 & DI.SOL & DNJY8301_mng_fdm.xIsx \\
\hline \multirow[t]{4}{*}{ Denmark, Jyndevad } & 1990 & All & DIJY9001.WTH & DIJY000002 & DI.SOL & DNJY9001_mng_fdm.xlsx \\
\hline & 1991 & All & DIJY9101.WTH & DIJY000002 & DI.SOL & DNJY9101_mng_fdm.xlsx \\
\hline & 1992 & All & DIJY9201.WTH & DIJY000002 & DI.SOL & DNJY9201_mng_fdm.xlsx \\
\hline & 1993 & All & DIJY9301.WTH & DIJY000002 & DI.SOL & DNJY9301_mng_fdm.xlsx \\
\hline \multirow[t]{3}{*}{ Denmark, Jyndevad } & 1984 & All & DIJY8401.WTH & DIJY000002 & DI.SOL & DNJY8401_mng_fdm.xlsx \\
\hline & 1985 & All & DIJY8501.WTH & DIJY000002 & DI.SOL & DNJY8501_mng_fdm.xIsx \\
\hline & 1986 & All & DIJY8601.WTH & DIJY000002 & DI.SOL & DNJY8601_mng_fdm.xlsx \\
\hline \multirow[t]{3}{*}{ Denmark, Tylstrup } & 1981 & All & DITY8101.WTH & DITY000002 & DI.SOL & DNTY8101_mng_fdm.xlsx \\
\hline & 1982 & All & DITY8201.WTH & DITY000002 & DI.SOL & DNTY8201_mng_fdm.xIsx \\
\hline & 1983 & All & DITY8301.WTH & DITY000002 & DI.SOL & DNTY8301_mng_fdm.xIsx \\
\hline Ecuador, San Gabriel & 1985 & All & CISG8501.WTH & IB00000009 & CH.SOL & IPSG8501_mng_fdm.xlsx \\
\hline \multirow[t]{5}{*}{ Finland, Jokioinen } & 1998 & OTC_AA_AA_98 & FI019801.WTH & FIFI0000019 & CH.SOL & CHFI9801_mng_fdm.xlsx \\
\hline & 1998 & OTC_NF_NF_98 & FI029801.WTH & FIFI0000019 & FI.SOL & CHFI9801_mng_fdm.xlsx \\
\hline & 1999 & OTC_NF+_NF_99 & FI039901.WTH & FIFI0000019 & FI.SOL & CHFI9801_mng_fdm.xlsx \\
\hline & 1999 & OTC_AA_AA_- 99 & FI049901.WTH & FIFI0000019 & FI.SOL & CHFI9801_mng_fdm.xlsx \\
\hline & 1999 & OTC_NF_NF_99 & FI039901.WTH & FIFI0000019 & FI.SOL & CHFI9801_mng_fdm.xlsx \\
\hline \multirow[t]{5}{*}{ Germany, Giessen } & 1998 & OTC_NF_CF_98 & GR039801.WTH & GRGR0000019 & GR.SOL & CHGR9801_mng_fdm.xlsx \\
\hline & 1998 & OTC_NF+_CF_98 & GR039801.WTH & GRGR0000019 & GR.SOL & CHGR9801_mng_fdm.xlsx \\
\hline & 1998 & OTC_NF++_CF_98 & GR039801.WTH & GRGR0000019 & GR.SOL & CHGR9801_mng_fdm.xIsx \\
\hline & 1998 & FACE_FACE_CF_98 & GR059801.WTH & GRGR0000019 & GR.SOL & CHGR9801_mng_fdm.xlsx \\
\hline & 1998 & FACE_FACE+_CF_98 & GR059801.WTH & GRGR0000019 & GR.SOL & CHGR9801_mng_fdm.xIsx \\
\hline \multirow[t]{5}{*}{ Germany, Giessen } & 1999 & OTC_NF_CF_99 & GR049901.WTH & GRGR0000019 & GR.SOL & CHGR9801_mng_fdm.xlsx \\
\hline & 1999 & OTC_NF+_CF_99 & GR049901.WTH & GRGR0000019 & GR.SOL & CHGR9801_mng_fdm.xIsx \\
\hline & 1999 & OTC_NF++_CF_99 & GR049901.WTH & GRGR0000019 & GR.SOL & CHGR9801_mng_fdm.xIsx \\
\hline & 1999 & FACE_FACE_CF_99 & GR069901.WTH & GRGR0000019 & GR.SOL & CHGR9801_mng_fdm.xlsx \\
\hline & 1999 & FACE_FACE+_CF_99 & GR069901.WTH & GRGR0000019 & GR.SOL & CHGR9801_mng_fdm.xlsx \\
\hline \multirow[t]{2}{*}{ India, Ludhiana } & 2008 & All & PULU0801.WTH & PLLU000001 & PL.SOL & PULU0801_mng_fdm.xlsx \\
\hline & 2010 & All & PULU0901.WTH & PLLU000001 ${ }^{\circ}$ & PL.SOL & PULU0901_mng_fdm.xlsx \\
\hline \multirow[t]{6}{*}{ India, Modipuran } & 2002 & All & CIMO0201.WTH & Cl_CLAF111 ${ }^{\circ}$ & Cl.SOL & CIMO0201_mng_fdm.xlsx \\
\hline & 2003 & All & CIMO0301.WTH & Cl_CLAF $111^{\circ}$ & CI.SOL & CIMO0301_mng_fdm.xIsx \\
\hline & 2004 & All & CIMO0401.WTH & Cl_CLAF111 ${ }^{\circ}$ & Cl.SOL & CIMO0401_mng_fdm.xlsx \\
\hline & 2005 & All & CIMO0501.WTH & $\mathrm{Cl} \_\mathrm{CLAF} 111^{\circ}$ & Cl.SOL & CIMO0501_mng_fdm.xIsx \\
\hline & 2006 & All & CIMO0601.WTH & Cl_CLAF111 ${ }^{\circ}$ & Cl.SOL & CIMO0601_mng_fdm.xlsx \\
\hline & 2007 & All & CIMO0701.WTH & Cl_CLAF111 ${ }^{\circ}$ & Cl.SOL & CIMO0701_mng_fdm.xlsx \\
\hline
\end{tabular}


Raymundo et al. 2018, Open Data Journal for Agricultural Research, vol. 4, pg. 35-44

Table 3. Continued

\begin{tabular}{|c|c|c|c|c|c|c|}
\hline Location & Year & Treatment $^{\mathrm{a}}$ & Weather File & Soil Profile & Soil File & $\begin{array}{r}\text { Management/measurement } \\
\text { File }\end{array}$ \\
\hline \multirow[t]{2}{*}{ India, Modipuran } & 2008 & All & CIMO0801.WTH & Cl_CLAF111 ${ }^{\circ}$ & CI.SOL & CIMO0801_mng_fdm.xlsx \\
\hline & 2009 & All & CIMO0901.WTH & Cl_CLAF111 ${ }^{\circ}$ & CI.SOL & CIMO0901_mng_fdm.xIsx \\
\hline \multirow[t]{5}{*}{ Ireland, Carlow } & 1998 & OTC_NF_NF_98 & IE019801.WTH & IEIE0000019 & IE.SOL & CHIE9801_mng_fdm.xIsx \\
\hline & 1998 & OTC_NF++_NF_98 & IE019801.WTH & IEIE0000019 & IE.SOL & CHIE9801_mng_fdm.xIsx \\
\hline & 1999 & OTC_NF_NF_99 & IECA9901.WTH & IEIE0000019 & IE.SOL & CHIE9801_mng_fdm.xIsx \\
\hline & 1999 & OTC_NF++_NF_99 & IECA9901.WTH & IEIE0000019 & IE.SOL & CHIE9801_mng_fdm.xIsx \\
\hline & 1999 & AA_99 & IE039901.WTH & IEIE0000019 & IE.SOL & CHIE9801_mng_fdm.xIsx \\
\hline \multirow[t]{2}{*}{ Italy, Rapolano } & 1998 & All & IT019702.WTH & ITIT0000019 & IT.SOL & CHRA9801_mng_fdm.xlsx \\
\hline & 1999 & All & IT019802.WTH & ITIT0000019 & IT.SOL & CHRA9801_mng_fdm.xIsx \\
\hline \multirow[t]{8}{*}{ Peru, La Molina } & 2003 & All & CILM0301.WTH & Cl_FLYE13A ${ }^{\circ}$ & Cl.SOL & CILM0301_mng_fdm.xlsx \\
\hline & 2004 & All & CILM0401.WTH & Cl_FLYE13A ${ }^{\circ}$ & CI.SOL & CILM0401_mng_fdm.xlsx \\
\hline & 2005 & All & CILM0501.WTH & CI_FLYE13A ${ }^{\circ}$ & CI.SOL & CILM0501_mng_fdm.xIsx \\
\hline & 2006 & All & CILM0601.WTH & Cl_FLYE13A ${ }^{\circ}$ & CI.SOL & CILM0601_mng_fdm.xlsx \\
\hline & 2007 & All & CILM0701.WTH & Cl_FLYE13A ${ }^{\circ}$ & Cl.SOL & CILM0701_mng_fdm.xlsx \\
\hline & 2008 & All & CILM0801.WTH & Cl_FLYE13A ${ }^{\circ}$ & Cl.SOL & CILM0801_mng_fdm.xIsx \\
\hline & 2009 & All & CILM0901.WTH & CI_FLYE13A ${ }^{\circ}$ & Cl.SOL & CILM0901_mng_fdm.xIsx \\
\hline & 2010 & All & CILM1001.WTH & Cl_FLYE13A ${ }^{\circ}$ & Cl.SOL & CILM1001_mng_fdm.xlsx \\
\hline Peru, La Molina & 2013 & All & CPLI9301.WTH & Cl_FLYE13A ${ }^{\circ}$ & CI.SOL & CPLI9301_mng_fdm.xIsx \\
\hline Peru, La Molina & 1985 & All & CPLM8501.WTH & LMCP970004º $^{\circ}$ & LM.SOL & CPLM8501_mng_fdm.xlsx \\
\hline Peru, San Ramon & 1984 & All & CPSR8401.WTH & SRUA85000 $1^{\circ}$ & SR.SOL & CPSR8401_mng_fdm.xIsx \\
\hline Peru, San Ramon & 2013 & All & CPLM9301.WTH & CPSR000022 & CH.SOL & CPSR9301_mng_fdm.xlsx \\
\hline \multirow[t]{2}{*}{ Scotland, Dundee } & 1984 & All & SCDU8401.WTH & SCDU000001 ${ }^{\mathrm{e}}$ & SC.SOL & SCDU8401_mng_fdm.xIsx \\
\hline & 1985 & All & SCDU8501.WTH & SCDU000002 & SC.SOL & SCDU8401_mng_fdm.xIsx \\
\hline \multirow[t]{2}{*}{ Scotland, Dundee } & 1986 & All & SCDU8601.WTH & SCDU000003 & SC.SOL & SCDU8401_mng_fdm.xlsx \\
\hline & 1987 & All & SCDU8701.WTH & SCDU000003 & SC.SOL & SCDU8401_mng_fdm.xlsx \\
\hline \multirow[t]{3}{*}{ Sweden, Gotenborg } & 1998 & OTC_NF++_NF_98 & SE019801.WTH & SESE0000019 & SE.SOL & CHSE9801_mng_fdm.xIsx \\
\hline & 1998 & OTC_NF_NF_98 & SE019801.WTH & SESE0000019 & SE.SOL & CHSE9801_mng_fdm.xlsx \\
\hline & 1998 & AA_98 & SE029801.WTH & SESE0000019 & SE.SOL & CHSE9801_mng_fdm.xlsx \\
\hline \multirow[t]{4}{*}{ Uganda, Kalengyere } & 2004 & All & UGKA0401.WTH & Cl_FRUG222 ${ }^{\circ}$ & CI.SOL & CIKA0401_mng_fdm.xIsx \\
\hline & 2005 & All & UGKA0501.WTH & Cl_FRUG $222^{\circ}$ & CI.SOL & CIKA0501_mng_fdm.xlsx \\
\hline & 2006 & All & UGKA0601.WTH & $\mathrm{Cl}$ FRUG222 ${ }^{\circ}$ & CI.SOL & CIKA0601_mng_fdm.xlsx \\
\hline & 2009 & All & UGKA0901.WTH & Cl_FRUG222 ${ }^{\circ}$ & CI.SOL & CIKA0901_mng_fdm.xIsx \\
\hline \multirow[t]{8}{*}{ United Kingdom, Sutton } & 1998 & OTC_NF_NF_98 & UK019801.WTH & UKUK0000019 & UK.SOL & CHUK9801_mng_fdm.xIsx \\
\hline & 1998 & OTC_NF+_NF_98 & UK019801.WTH & UKUK0000019 & UK.SOL & CHUK9801_mng_fdm.xlsx \\
\hline & 1998 & OTC_NF++_NF_98 & UK019801.WTH & UKUK000001 ${ }^{\mathrm{g}}$ & UK.SOL & CHUK9801_mng_fdm.xIsx \\
\hline & 1998 & AA_98 & UK029801.WTH & UKUK000001 & UK.SOL & CHUK9801_mng_fdm.xIsx \\
\hline & 1999 & OTC_NF_NF_99 & UK039901.WTH & UKUK0000019 & UK.SOL & CHUK9801_mng_fdm.xlsx \\
\hline & 1999 & OTC_NF+_NF_99 & UK039901.WTH & UKUK0000019 & UK.SOL & CHUK9801_mng_fdm.xlsx \\
\hline & 1999 & OTC_NF++_NF_99 & UK039901.WTH & UKUK0000019 & UK.SOL & CHUK9801_mng_fdm.xIsx \\
\hline & 1999 & AA_99 & UK049901.WTH & UKUK000001 ${ }^{\mathrm{g}}$ & UK.SOL & CHUK9801_mng_fdm.xlsx \\
\hline United States, Benton & 2003 & All $^{-}$ & USBE0301.WTH & USBE000001 & US.SOL & USBE0301_mng_fdm.xIsx \\
\hline United States, Hastings & 2011 & All & UFHA1101.WTH & UHHA0000049 & UH.SOL & UFHA1101_mng_fdm.xIsx \\
\hline United States, New York & 1980 & All & NYCU8001.WTH & IBPT910006 & SOIL.SOL & NYCU8001_mng_fdm.xlsx \\
\hline $\begin{array}{l}\text { United States, Idaho } \\
\text { (Oregon) }\end{array}$ & 1988 & All & OSBO8801.WTH & IBPT910005 & SOIL.SOL & OSBO8801_mng_fdm.xlsx \\
\hline \multirow[t]{4}{*}{ United States, Suwanee } & 2010 & All & NASA1001.WTH & SF00000001 ${ }^{\circ}$ & SF.SOL & SFUF1001_mng_fdm.xIsx \\
\hline & 2011 & All & NASA1101.WTH & SF00000002 ${ }^{\circ}$ & SF.SOL & SFUF1001_mng_fdm.xlsx \\
\hline & 2012 & All & NASA1201.WTH & SF00000003 ${ }^{\circ}$ & SF.SOL & SFUF1001_mng_fdm.xlsx \\
\hline & 2013 & All & NASA1301.WTH & SF00000004 ${ }^{\circ}$ & SF.SOL & SFUF1001_mng_fdm.xlsx \\
\hline United States, Suwanee & 2001-2003 & All & SF0110004.WTH & NFSF000001 & NF.SOL & SF011000_mng_fdm.xlsx \\
\hline
\end{tabular}

a Treatments were indicated when weather data or soil profile varied

All: Treatments used the same weather data and/or soil profile

NF_NF: $\quad$ OTC or FACE ring non treated.

$\mathrm{NF}+\mathrm{NF}: \quad$ OTC or FACE ring enriched with $\mathrm{CO}_{2}(550 \mathrm{ppm})$.

$\mathrm{NF++} N \mathrm{NF}: \quad$ OTC enriched with $\mathrm{CO}_{2}(680 \mathrm{ppm})$.

AA: - Ambient air plot.

e Soil profile computed with SBuild soil parameter estimation tool available in the DSSAT-CSM (Raymundo et al., 2017).

${ }^{9}$ Soil profile derived from soil texture (Raymundo et al., 2017).

${ }^{\circ}$ Soil profile from publications (see Table 1). 
2 ACKNOWLEDGMENTS We thank the International Research Food Policy Research Institute (IFPRI), the International Potato Center (CIP), and the USAID linkage fund project "Collaboration between the International Potato Center (CIP) and the University of Florida (UF) to better understand and prioritize climate change adaptation needs for food security in the Andes." We also thank Cheryl Porter and Meng Zhang, University of Florida, for assistance with AgMIP data formats.

\section{REFERENCES}

Albert, M., 2002. Monitoring and modeling the fate and transport of nitrate in the vadose zone beneath a Suwanneee River Basin vegetable farm. Department of Agricultural and Biological Engineering. University of Florida, Gainesville, Florida, pp. 1-179.

Alva, A.K., Marcos, J., Stockle, C., Reddy, V.R., Timlin, D., 2010. A crop simulation model for predicting yield and fate of nitrogen in irrigated potato rotation cropping system. Journal of Crop Improvement 24, 142-152. doi: 10.1080/15427520903581239.

Arora, V.K., Nath, J.C., Singh, C.B., 2013. Analyzing potato response to irrigation and nitrogen regimes in a sub-tropical environment using SUBSTOR-Potato model. Agricultural Water Management 124, 69-76. doi: 10.1016/i.agwat.2013.03.021.

Bacastow, R.B., Keeling, C.D., Whorf, T.P., 1985. Seasonal amplitude increase in atmospheric $\mathrm{CO}_{2}$ concentration at Mauna Loa, Hawaii,1959-1982. Journal of Geophysical Research-Atmospheres 90, 10529-10540. doi: https://doi.org/10.1029/JD090iD06p10529.

Bach, A., Nielsen, S., 1985. Vækstanalyse i kartofler 1981-83. Planteavl 89, 215-224.

Clavijo Ponce, N.L., 1999. Validación del modelo de simulacion DSSAT en el cultivo de papa (Solanum tuberosum L.) en las condiciones del canton Montufar provincia del Carchi. Escuela de ingenieria agronómica. Escuela superior politécnica de Chimborazo, Riobamba, Ecuador, p. 80.

Condori, B., Hijmans, R.J., Quiroz, R., Ledent, J.F., 2010. Quantifying the expression of potato genetic diversity in the high Andes through growth analysis and modeling. Field Crops Research 119, 135-144. doi: 10.1016/j.fcr.2010.07.003.

De Temmerman, L., Hacour, A., Guns, M., 2002. Changing climate and potential impacts on potato yield and quality 'CHIP': introduction, aims and methodology. European Journal of Agronomy 17, 233242. doi: $10.1016 / \mathrm{S} 1161-0301(02) 00063-1$

Edlefsen, O., 1991. Styring af kvælstof i vandede kartofler. The Danish Institute of Agricultural Science, Tjele, Denmark.

Forero Hernandez, D., Garzon Montaño, E., 2000. Validacion del modelo de simulacion de crecimiento "SUBSTOR-potato V.35" para cuatro variedades mejoradas de papa (Solanum tuberosum ssp. andigena) bajo condiciones de cultivo comercial. In: Montano, E.G. (Ed.), Facultad De Agronomia. Universidad Nacional De Colombia, Bogota, pp. 1-84.

Gao, J.L., Liu, K.L., Sheng, J.H., Ren, K., Wen, X.J., Sui, Q.J., Jiang, B., 2004. Dry matter accumulation and distribution of potato under dry farming. Potato China 18, 9-15.

Gao, J.L., Liu, K.L., Zhang, B.L., Ren, Y.Z., 2003. Accumulation and distribution of dry matter in potato. China Potato 17, 209-212.

Hoogenboom, G., Jones, J.W., Wilkens, P.W., Porter, C.H., Boote, K.J., Hunt, L.A., Singh, U., Lizaso, J.L., White, J.W., Uryasev, O., Royce, F.S., Ogoshi, R., Gijsman, A.J., Tsuji, G.Y., Koo, J., 2015. Decision Support System for Agrotechnology Transfer (DSSAT) Version 4.6 [CD-ROM]. University of Hawaii, Honolulu, Hawaii.

Jørgensen, V., 1984. Vandforsyningens indflydelse på udbytte og kvalitet af kartofler. Planteavl 88, 453468.

Jørgensen, V., Edlefsen, O., 1987. Vandforsyningens indflydelse på udbytte og kvalitet af industrikartofler. Planteavl 91, 329-347.

Kleinwechter, U., Gastelo, M., Ritchie, J., Nelson, G., Asseng, S., 2016. Simulating cultivar variations in potato yields for contrasting environments. Agricultural Systems 145, 51-63. doi: 10.1016/i.agsy.2016.02.011.

Liu, K.L., Gao, J.L., Ren, K., Sheng, J.H., Sui, Q.J., Jiang, B., 2003a. Nitrogen absorption, accumulation and distribution of potato under dry farming. Potato China 17, 321-325.

Liu, K.L., Gao, J.L., Sun, H.Z., Sheng, J.H., 2003b. The dynamic of sink structure in potato. China Potato $17,267-272$.

Marshall, B., Van Den Broek, B.J., 1995. Field experiments and analysis of data used in the case study. Modelling and parameterization of the soil-plant-atmosphere system: A comparison of potato growth models, 179-210. url: http://library.wur.nl/WebQuery/wurpubs/302080. 
Nelson, D.G., 1987. Light interception, dry matter production and partitioning of the potato crop in tropical environments. Department of Agriculture. University College of Wales, pp. 1-135.

Prasad, R., Hochmuth, G.J., Boote, K.J., 2015. Estimation of Nitrogen Pools in Irrigated Potato Production on Sandy Soil Using the Model SUBSTOR. PloS one 10, e0117891-e0117891. doi: 10.1371/journal.pone.0117891.

Raymundo, R., Asseng, S., Prassad, R., Kleinwechter, U., Concha, J., Condori, B., Bowen, W., Wolf, J., Olesen, J.E., Dong, Q.X., Zotarelli, L., Gastelo, M., Alva, A., Travasso, M., Quiroz, R., Arora, V., Graham, W., Porter, C., 2017. Performance of the SUBSTOR-potato model across contrasting growing conditions. Field Crops Research 202, 57-76. doi: 10.1016/i.fcr.2016.04.012.

Travasso, M.I., Caldiz, D.O., Saluzzo, J.A., 1996. Yield prediction using the SUBSTOR-potato model under Argentinian conditions. Potato Research 39, 305-312. doi: 10.1007/BF02360922.

Trebejo, I., Midmore, D.J., 1990. Effect of water stress on potato growth, yield and water use in a hot and a cool tropical climate. The Journal of Agricultural Science 114, 321-334. doi: 10.1017/S0021859600072713.

Warren, M.F., 2003. Monitoring and modeling water and nitrogen transport in the vadose zone of a vegetable farm in the Suwannee river basin. Department of Agricultural and Biological Engineering. University of Florida, Gainesville, Florida, pp. 1-233.

White, J.W., Hunt, L.A., Boote, K.J., Jones, J.W., Koo, J., Kim, S., Porter, C.H., Wilkens, P.W., Hoogenboom, G., 2013. Integrated description of agricultural field experiments and production: The ICASA Version 2.0 data standards. Computers and Electronics in Agriculture 96, 1-12. doi: 10.1016/i.compag.2013.04.003.

Zotarelli, L., Rens, L.R., Cantliffe, D.J., Stoffella, P.J., Gergela, D., Fourman, D., 2014. Nitrogen Fertilizer Rate and Application Timing for Chipping Potato Cultivar Atlantic. Agronomy Journal 106, 22152226. doi: $10.2134 /$ agroni14.0193. 